\title{
Epithelial-mesenchymal transition in gingival tissues from chronic periodontitis patients: A case-control study
}

\author{
Karim Wasfi Wadie ${ }^{1, A-F}$, Maha Hassan Bashir ${ }^{2, A, E, F}$, Marwa Magdy Saad Abbass ${ }^{2, A, C, E, F}$ \\ ${ }^{1}$ Department of Oral Biology, Faculty of Dentistry, Modern University for Technology and Information, Cairo, Egypt \\ ${ }^{2}$ Department of Oral Biology, Faculty of Dentistry, Cairo University, Egypt \\ A - research concept and design; $\mathrm{B}$ - collection and/or assembly of data; $\mathrm{C}$ - data analysis and interpretation; \\ $D$ - writing the article; $E$ - critical revision of the article; $F$ - final approval of the article
}

Address for correspondence

Karim Wasfi Wadie

E-mail: karimwasfi3@gmail.com

Funding sources

None declared

Conflict of interest

None declared

Acknowledgements

Karim Wasfi Wadie would like to express his gratitude to

Dr. Marwa Magdy Saad Abbass and Dr. Maha Hassan Bashirfor their unfailing support and assistance during his Master's research. He is also grateful to his parents and sister who have provided him with moral and emotional support in his life, and to his friends who have supported him along the way. Many thanks to you for all your encouragement.

Received on 0ctober 1, 2020

Reviewed on February 12, 2021

Accepted on February 20, 2021

Published online on September 30, 2021

Cite as

Wadie KW, Bashir MH, Abbass MMS. Epithelial-mesenchymal transition in gingival tissues from chronic periodontitis patients: A case-control study. Dent Med Probl. 2021;58(3):311-319. doi:10.17219/dmp/133514

DOI

$10.17219 / \mathrm{dmp} / 133514$

Copyright

( 2021 by Wroclaw Medical University

This is an article distributed under the terms of the

Creative Commons Attribution 3.0 Unported License (CC BY 3.0)

(https://creativecommons.org/licenses/by/3.0/).

\section{Abstract}

Background. It has been proposed that epithelial-mesenchymal transition (EMT) is responsible for the pathogenesis of several diseases. However, the relationship between the EMT process and the severity of periodontitis has not been previously investigated.

Objectives. This study aimed to localize and quantitatively assess the expression of transforming growth factor-beta 1 (TGF- $\beta 1$ ), vimentin and E-cadherin in correlation with the EMT process in human gingiva of periodontally diseased patients in comparison with halthy individuals.

Material and methods. Gingival tissue samples from 36 participants were divided into 2 groups: the healthy (control) group $(n=9)$; and the periodontitis group $(n=27)$. The periodontitis group was further subclassified into mild, moderate and severe periodontitis subgroups (9 patients in each subgroup). The samples were subjected to histological staining, the histomorphometric analysis and the quantitative real-time polymerase chain reaction (RT-PCR) analysis for TGF- $\beta$, vimentin and E-catherin. Statistical and correlation analyses were performed.

Results. The hematoxylin and eosin (H\&E) stain sections from both the moderate and severe periodontitis subgroups showed epithelial hyperplasia, perinuclear haloing and a marked increase in the inflammatory cell count as compared to the control group. The highest mean TGF- $\beta 1$ and vimentin expression values were recorded in the severe periodontitis subgroup, whereas the lowest mean values were recorded in the control gingiva. On the contrary, the expression of E-catherin had the highest mean value in the control gingiva, whereas the lowest mean value was recorded in the severe periodontitis subgroup. All results were found to be statistically significant. The correlation analysis revealed a statistically significant positive correlation between the severity of periodontitis and the expression of TGF- $\beta$ and vimentin, while a statistically significant inverse correlation was found between the expression of $\mathrm{E}$-catherin and the severity of periodontitis.

Conclusions. There is a direct correlation between the severity of periodontitis and the expression of the EMT process markers (TGF- $\beta$ and vimentin). This correlation indicates that EMT plays an important role in the pathogenesis and prognosis of periodontal disease. The data presented in this study could open the door for using anti-EMT agents in treating periodontal disease.

Keywords: periodontitis, transforming growth factor-beta, vimentin, E-cadherin, epithelial-mesenchymal transition 


\section{Introduction}

Periodontitis is an epidemic disease that affects a high percentage of the population. According to a systematic review from 2018, an estimated $42 \%$ of dentate US adults at the age of 30 years or older had periodontitis, with $7.8 \%$ having severe periodontitis; severe periodontitis was most prevalent among adults aged 65 years or older, Mexican Americans, non-Hispanic Black Americans, and smokers. ${ }^{1}$ A study published in 2019 found that in Egypt, the prevalence of periodontitis was 89.8\%; 70.8\% of participants had stage I and $15.2 \%$ had stage II, while only $4.4 \%$ and $2.1 \%$ suffered from stage III and stage IV, respectively. ${ }^{2}$

Periodontitis encompasses a group of inflammatory conditions that affect the supporting tissues of the dentition. The disease is characterized by an aberrant host response in susceptible patients to the plaque biofilm, which consists of Gram-negative anaerobic bacteria. ${ }^{3}$ Hernández et al. reported that these pathogens could stimulate host cells, including the junctional epithelium (which is closely related to the plaque biofilm), to secrete a range of pro-inflammatory cytokines involved in initiating the epithelial-mesenchymal transition (EMT) process. ${ }^{4}$ It has been proposed that the EMT process and the resulting shift from an epithelial to mesenchymal phenotype could result in the loss of the integrity of the epithelial barrier, which would allow bacterial invasion into the underlying tissues and predispose the patient to periodontal disease. ${ }^{3}$

Epithelial-mesenchymal transition is a biological process that causes the polarized epithelial cells to undergo multiple biochemical changes to assume a mesenchymal cell phenotype. ${ }^{5}$ There is growing evidence that EMT plays important roles in embryogenesis, inflammation and cancer metastasis. A series of distinct molecular events appears to be involved in triggering EMT, such as the activation of certain transcriptional pathways, alterations in the expression of surface molecules and changes in cytoskeletal proteins. ${ }^{6}$ Moreover, cytokines and bacterial products have been highlighted as EMT-predisposing factors. ${ }^{7}$

The loss of cell-cell adhesion, and the downregulation of E-cadherin and other epithelial markers (occludin, laminin-1, matrix metallopeptidase-9 (MMP-9), and integrin) are the principal characteristics of EMT. These changes can occur in concert with the upregulation of key transcription factors and the expression of associated mesenchymal markers, such as vimentin, $\mathrm{N}$-cadherin, fibronectin, and laminin $-5 .^{8}$

This study aimed to compare the expression of transforming growth factor-beta 1 (TGF- $\beta 1$ ), vimentin and E-cadherin in the gingival tissue samples obtained from periodontally diseased patients and in the tissue samples taken from individuals with healthy gingiva.

\section{Material and methods}

\section{Sample size}

Based on a previous study by Arora et al., which stated that the expression of E-cadherin among controls was 99\% and the expression for periodontitis was 44\%, using a power of $80 \%$ and a significance level of $5 \%,{ }^{9} 9$ samples in each group were considered sufficient. The sample size was calculated using the PS: Power and Sample Size Calculation software, v. 3.1.2 (Vanderbilt University, Nashville, USA; http://ps-power-and-sample-size-calculation. freedownloadscenter.com/windows/).

\section{Subjects and sample collection}

This study was conducted according to the regulations of the Ethics Committee of the Faculty of Dentistry at Cairo University in Egypt. The research was approved by the institutional ethics committee, and written informed consent was obtained from all of the participants involved in the study. A total of 36 participants were recruited.

The participants were divided according to their periodontal status into 2 main groups: the control group $(n=9)$; and the periodontitis group $(n=27)$, which consisted of 3 subgroups (mild, moderate and severe periodontitis) with 9 patients in each subgroup. The patients were classified into the mild, moderate and severe periodontitis subgroups based on the collected clinical data, according to the American Academy of Periodontology criteria from $2015 .^{10}$

The study was performed on the gingival tissue samples obtained from both chronic periodontitis patients and healthy individuals. All samples were kindly provided by donors from the outpatient clinics at the Faculty of Dentistry, Cairo University. The inclusion criteria for the healthy group were as follows: the absence of any clinical signs of gingival inflammation; probing depth $(P D) \leq 3 \mathrm{~mm}$; and no clinical attachment loss (CAL). The inclusion criteria for the periodontitis group comprised a generalized presence of clinical signs of gingival inflammation, generalized $\mathrm{PD} \geq 4 \mathrm{~mm}$ and generalized $\mathrm{CAL} \geq 2 \mathrm{~mm}$. Generalized chronic periodontitis is defined as periodontitis without a clear pattern of disease distribution in the affected teeth, with more than $30 \%$ of the teeth affected. ${ }^{11}$ The exclusion criteria were the following: patients with any systemic diseases; smokers; pregnant or lactating women; patients with cervi$\mathrm{cal} /$ proximal/subgingival caries or restorations; and patients who received periodontal or antimicrobial therapy within 3 months before sampling. The gingival tissue samples were harvested from November 2017 until February 2018, during periodontal surgery and the extraction of periodontally hopeless teeth for the periodontitis patients, and during premolar extraction (orthodontic 
treatment) or third molar extraction for the healthy control patients. Each specimen from each experimental group was divided into halves; one half was used for the light microscopic examination and the histomorphometric analysis, while the other half was used for the gene expression analysis.

To minimize the number of variables during the study, potential confounders were excluded within the exclusion and inclusion criteria. In addition, to avoid human variations and remove bias, the sample preparation, cutting and staining were completed in the same laboratory by the same technician, according to standardized criteria. Moreover, to avoid selection, performance and detection bias, the patients were defined eligible based on consecutive order. Blinding was applied for the assessors of both the histological and quantitative real-time polymerase chain reaction (RT-PCR) results.

The flow chart of the study is presented in Fig. 1.

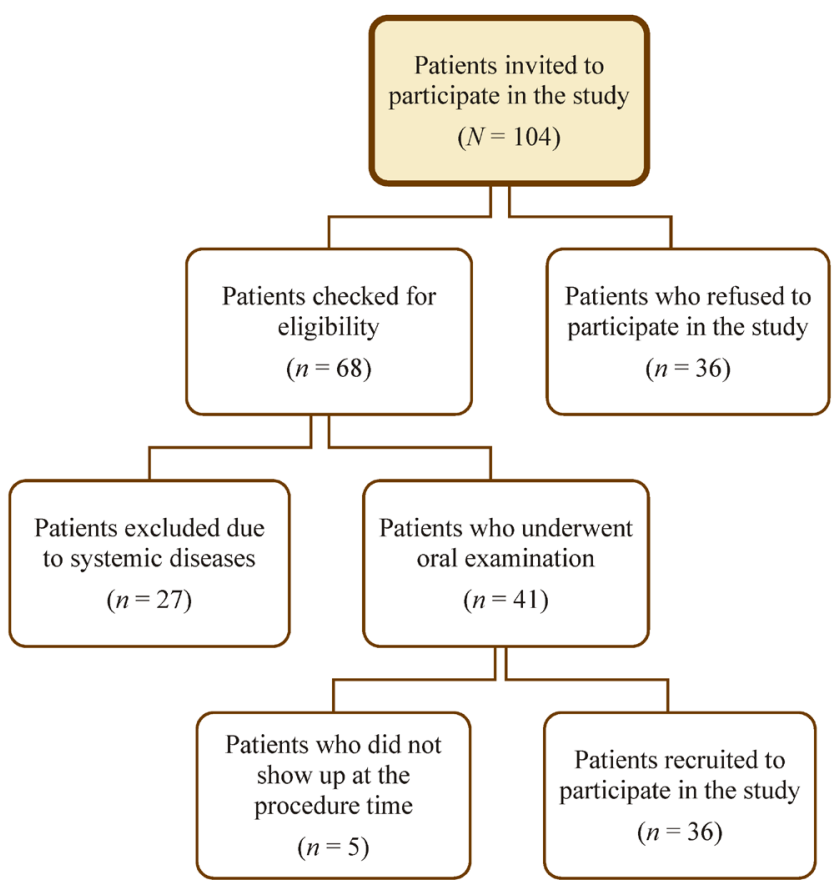

Fig. 1. Flow chart of the study

\section{Experimental procedure}

\section{Light microscopic examination}

The specimens were dehydrated in ascending grades of alcohol, then cleared in xylol and embedded in paraffin blocks. Sections of 4-5-micrometer thickness were mounted on ordinary glass slides and stained with hematoxylin and eosin (H\&E) (Abbey Color, Philadelphia, USA) for routine histological evaluation, according to the conventional method. ${ }^{12}$

\section{Histomorphometric analysis}

The image analysis was performed using a Leica microscope equipped with a digital video camera and software (Leica Qwin 500; Leica Microsystems, Wetzlar, Germany). The image analysis system was used to assess the epithelial thickness and to count the number of inflammatory cells per area unit in order to assess the degree of inflammation.

\section{Quantitative RT-PCR}

The specimens were used to measure the expression of TGF- $\beta 1$, vimentin and E-catherin. Total RNA was isolated using a tissue extraction kit (Qiagen Sciences Inc., Germantown, USA) according to the manufacturer's instructions. Total RNA $(0.5-2 \mu \mathrm{g})$ was used for cDNA conversion with a high-capacity cDNA reverse transcription kit (Fermentas, Waltham, USA). The quantitative RT-PCR amplification and analysis were performed using the StepOne ${ }^{\mathrm{TM}}$ system (Applied Biosystems, Waltham, USA). Relative quantification (RQ) was calculated using the Applied Biosystems software, v. 3.1 (Table 1).

Table 1. Primer sequences of the genes used in the study

\begin{tabular}{|l|c|c|} 
Protein name & $\begin{array}{c}\text { Gene } \\
\text { symbol }\end{array}$ & \multicolumn{1}{c}{ Primer sequence $\left(5^{\prime}-3^{\prime}\right)$} \\
TGF- $\beta 1$ & TGF- $\beta 1$ & $\begin{array}{l}\text { forward }- \text { GCAGCACGTGGAGCTGTA } \\
\text { reverse }- \text { CAGCCGGTTGTGAGGTA }\end{array}$ \\
Vimentin & VIM & $\begin{array}{l}\text { forward }- \text { CCCTCGCTCTCTTCTTGCAG } \\
\text { reverse }- \text { AATGACTGCAGGGTGCTCTC }\end{array}$ \\
E-cadherin & CDH1 & $\begin{array}{l}\text { forward }- \text { TTCTCCGCGCTCCTGCTCCT } \\
\text { reverse }- \text { TTGTCAGCTCCTGGGCCGGT }\end{array}$ \\
\hline
\end{tabular}

TGF- $\beta 1$ - transforming growth factor-beta 1.

\section{Reagents and equipment}

In the present study, the following reagents and equipment were used:

- Moloney murine leukemia virus reverse transcriptase (M-MLV RT) for the synthesis of cDNA from RNA;

- human placental ribonuclease inhibitor;

- first-strand buffer;

- deoxynucleotide triphosphates (dNTPs) - dATP, dTTP, dGTP, and dCTP;

- random hexamers - primers for the reverse transcription of RNA (Stratagene California, La Jolla, USA);

- diethyl pyrocarbonate (DEPC)-treated water; and

- a thermal cycler (Biometra ${ }^{\circledR}$; Analytik Jena, Jena, Germany). 


\section{Calculation of relative quantification (relative expression)}

Relative quantification was calculated according to the Applied Biosystems software using the following formula (Equation 1):

$$
\mathrm{RQ}=2^{-\Delta \Delta \mathrm{Ct}}
$$

where:

$\Delta \mathrm{Ct}=\mathrm{Ct}$ gene test $-\mathrm{Ct}$ endogenous control; $\Delta \Delta \mathrm{Ct}=\Delta \mathrm{Ct}$ treated sample $-\Delta \mathrm{Ct}$ calibrator; $\mathrm{Ct}$ - cycle threshold.

Relative quantification is a fold change as compared to the calibrator (untreated sample).

\section{Statistical analysis}

The data is presented as means $(M) \pm$ standard deviations $(S D)$. The data was first tested for normality with the Kolmogorov-Smirnov test, which revealed that most data was parametric. The one-way analysis of variance (ANOVA) was used for comparisons between the groups. This was followed by Tukey's post hoc test when ANOVA revealed a significant difference. The level of significance was established at $p<0.05$.

The analysis of correlation between various variables was performed using the Pearson product-moment correlation equation for the linear relation of normally distributed variables. Two-sided $p<0.05$ was considered statistically significant. All statistical calculations were performed with the use of the IBM SPSS Statistics for Windows software, v. 22.0 (IBM Corp., Armonk, USA).

The power analysis was performed for the only statistically insignificant variable. The mean value of the variable in each group was entered and the highest $S D$ was selected as an intragroup variation parameter. The effect size was calculated, and then entered to calculate the power of the statistical results. The omnibus one-way ANOVA test was used in the analysis with a type I error probability equal to 0.05 . The calculations were performed using the $G^{*}$ Power software v. 3.1.2 for Windows (https://www. psychologie.hhu.de/arbeitsgruppen/allgemeine-psychologie-und-arbeitspsychologie/gpower).

\section{Results}

\section{Light microscopic examination}

\section{Epithelium}

The gingival epithelium of the moderate and severe periodontitis subgroups showed epithelial hyperplasia, while that of the mild periodontitis subgroup exhibited a mild increase in the epithelial thickness as compared to the control group (Fig. 2). The epithelial ridges of the moderate and severe periodontitis subgroups showed elongation and a tortuous appearance. When examining the mild periodontitis subgroup, however, it was observed that the epithelial ridges had a configuration similar to that of the control group. The basement membrane was intact in both the control group and the mild periodontitis subgroup, while there was a loss of continuity in some areas in the moderate and severe periodontitis subgroups (Fig. 2).

The moderate and severe periodontitis subgroups showed migrating inflammatory cells that were scattered throughout the layers of the epithelium, but were mainly concentrated in the basal and parabasal cell layers. On the other hand, there were only a few of these cells in the epithelium of the mild periodontitis subgroup and the control group (Fig. 3).

Perinuclear haloing was observed in the basal and parabasal cell layers in both the moderate and severe periodontitis subgroups. Conversely, no similar haloing was observed in the basal and parabasal cell layers in the mild periodontitis subgroup and the control group (Fig. 3). Severe perinuclear haloing was observed in the prickle cell layers in the tissue samples from the moderate and severe periodontitis patients (Fig. 3). Haloing was also noted in the prickle cell layers in the samples from the mild periodontitis subgroup and the control group, but to a lesser extent (Fig. 4).

The flattening of the granular cell layer and the disappearance of its keratohyalin granules were detected in the gingiva from the severe periodontitis patients. The

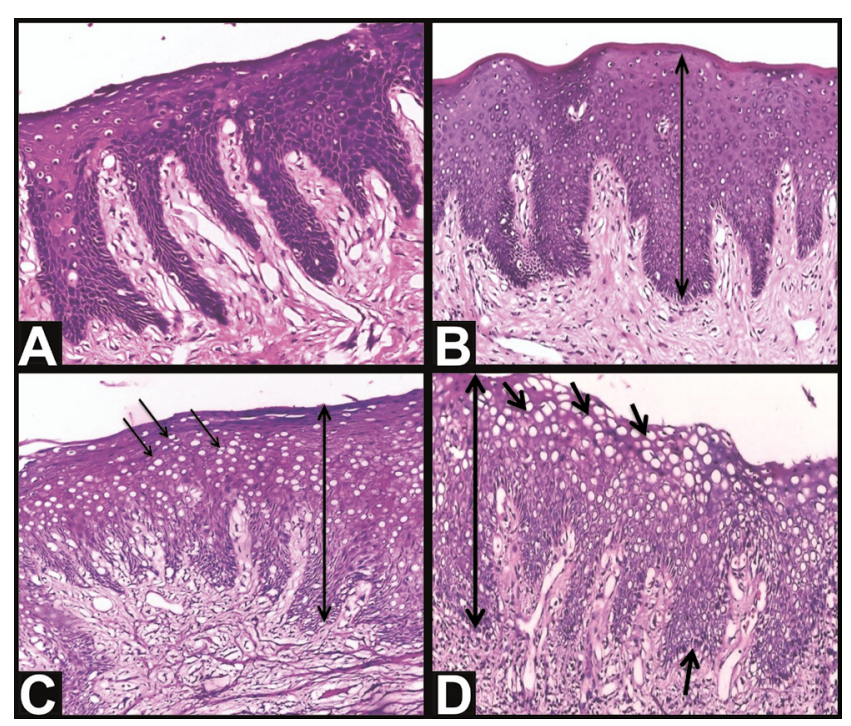

Fig. 2. Photomicrographs of the gingiva from the control group (A) and the mild periodontitis subgroup (B), showing the mild elongation (doublehead arrow) and normal configuration of the epithelial ridges, and of the moderate (C) and severe (D) periodontitis subgroups, showing epithelial hyperplasia, and the elongation (double-head arrows) and tortuous appearance of the epithelial ridges (single-head arrows)

Hematoxylin and eosin (H\&E) stain; $\times 40$ magnification. 


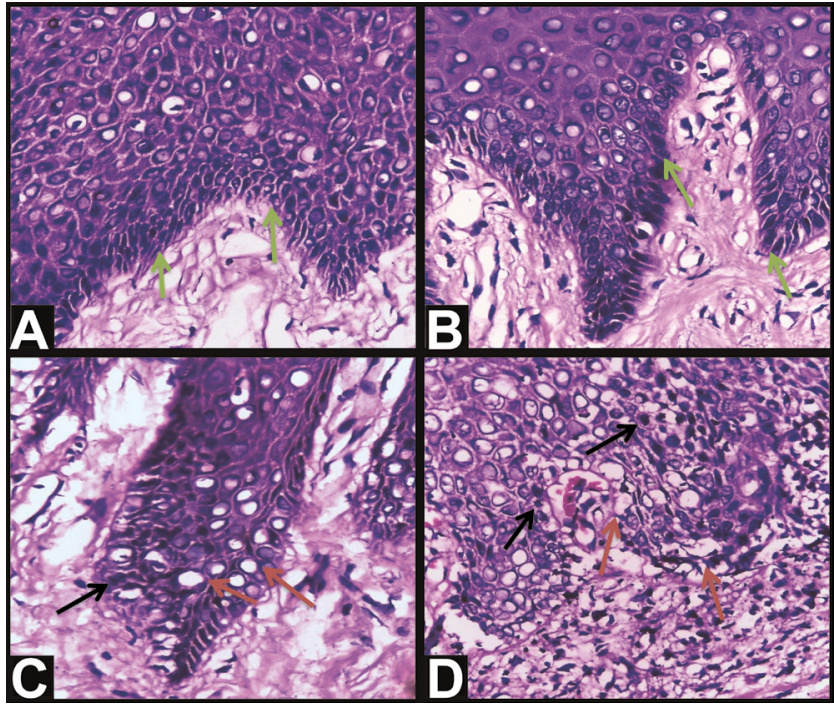

Fig. 3. Photomicrographs of the gingiva from the control group $(A)$ and the mild periodontitis subgroup (B), showing the basal and parabasal cell layers with the absence of perinuclear haloing and an intact basement membrane (green arrows), and of the moderate (C) and severe (D) periodontitis subgroups, showing migrating inflammatory cells (black arrows), perinuclear haloing in the basal, parabasal and prickle cell layers, and the loss of continuity of the basement membrane (red arrows)

H\&E stain; $\times 40$ magnification

gingiva from the moderate periodontitis subgroup also showed the disappearance of keratohyalin granules and perinuclear haloing in the granular cells. On the other hand, keratohyalin granules were present in the gingival tissues of the mild periodontitis subgroup and the control group (Fig. 4).

The gingival epithelium from the moderate and severe periodontitis subgroups showed a reduction of the keratin layer. There was also severe vacuolization,

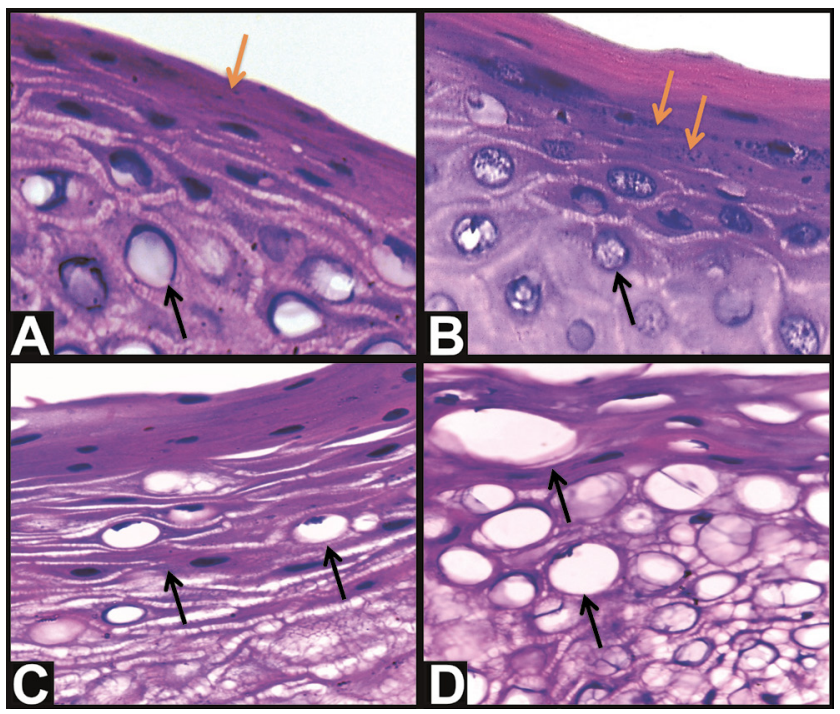

Fig. 4. Photomicrographs of the gingiva from the control group (A), and the mild (B), moderate (C) and severe (D) periodontitis subgroups, showing changes in the keratin layer and perinuclear haloing in the superficial and prickle cell layers (black arrows) with a gradual loss of keratohyalin granules (orange arrows)

H\&E stain; $\times 100$ magnification. and perinuclear haloing was present in the superficial cells. Conversely, the gingiva from the mild periodontitis subgroup showed that keratin was present, which was similar to what was observed in the control group (Fig. 4).

\section{Lamina propria}

The gingival tissues from all of the periodontitis patients showed inflammatory cell infiltration in their papillary and reticular layers at different grades, in proportion to the severity of periodontal inflammation. On the contrary, the samples from the control group showed very few inflammatory cells (Fig. 5).

The mild periodontitis subgroup showed few capillaries and dilated small blood vessels engorged with blood. In the moderate periodontitis subgroup there was a slight increase in the number of small capillaries and blood vessels, while the severe periodontitis subgroup showed a marked increase in vasculature (the formation of small capillaries) and few moderatesized blood vessels (Fig. 5).

\section{Histomorphometric analysis}

The highest mean epithelial thickness was recorded in the severe periodontitis subgroup $(178.348 \mu \mathrm{m})$, whereas the lowest mean value was recorded in the control group $(89.778 \mu \mathrm{m})$. The difference between the groups was statistically significant $(p<0.028)$. There were no significant differences between the control group and the mild and moderate periodontitis subgroups $(p>0.05)$ (Table 2).

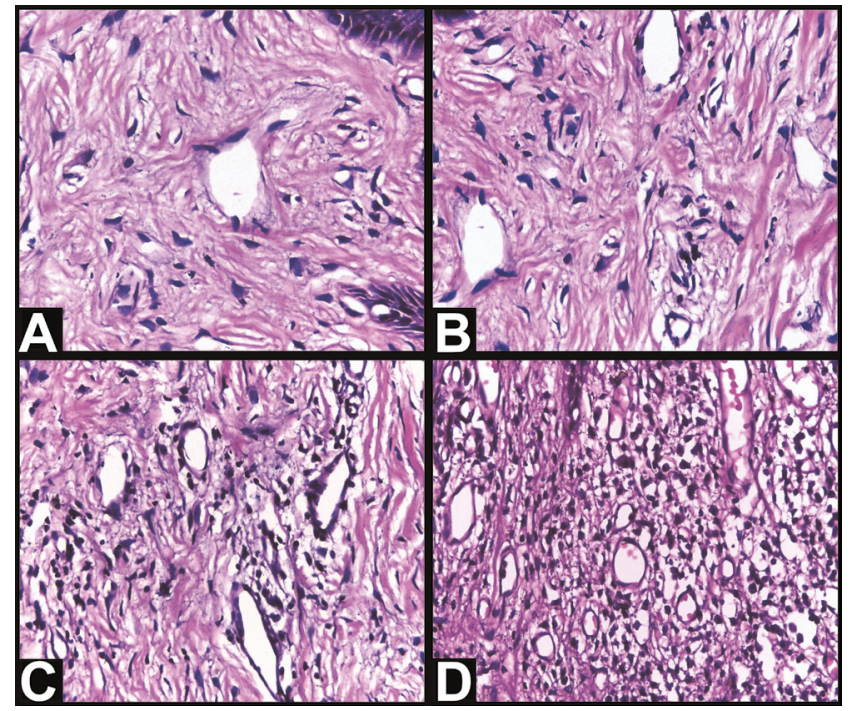

Fig. 5. Light micrographs of the connective tissue from the control group $(A)$, and the mild (B), moderate (C) and severe (D) periodontitis subgroups, showing differences in collagen fiber orientation, and a gradual increase in the blood vessel size and the number of inflammatory cells

H\&E stain; $\times 40$ magnification. 
Table 2. Descriptive statistics regarding the epithelial thickness and the inflammatory cell count, and comparisons between the control group and the periodontitis subgroups (ANOVA)

\begin{tabular}{|c|c|c|c|}
\hline Measured items & Groups & $M \pm S D$ & $p$-value \\
\hline \multirow{4}{*}{$\begin{array}{l}\text { Epithelial thickness } \\
{[\mu \mathrm{m}]}\end{array}$} & control group & $89.778^{b} \pm 20.546$ & \multirow{4}{*}{$<0.028^{*}$} \\
\hline & mild periodontitis subgroup & $101.391^{\mathrm{a}, \mathrm{b}} \pm 37.607$ & \\
\hline & moderate periodontitis subgroup & $145.763^{\mathrm{a}, \mathrm{b}} \pm 50.985$ & \\
\hline & severe periodontitis subgroup & $178.348^{\mathrm{a}} \pm 63.643$ & \\
\hline \multirow{4}{*}{$\begin{array}{l}\text { Inflammatory cell count } \\
\text { [cells/ } \mu \mathrm{L}]\end{array}$} & control group & $44,523 \pm 25,993$ & \multirow{4}{*}{$<0.177$} \\
\hline & mild periodontitis subgroup & $74,865 \pm 43,791$ & \\
\hline & moderate periodontitis subgroup & $95,414 \pm 61,621$ & \\
\hline & severe periodontitis subgroup & $123,313 \pm 74,124$ & \\
\hline
\end{tabular}

$M$ - mean; SD - standard deviation; ${ }^{*}$ statistically significant $(p<0.05)$; means sharing the same superscript letter within the same comparison are not significantly different.

The highest mean count of inflammatory cells was recorded in the severe periodontitis subgroup $(123,313$ cells $/ \mu \mathrm{L})$, whereas the lowest mean value was recorded in the control group $(44,523$ cells $/ \mu \mathrm{L})$. The ANOVA test revealed that the difference between the groups was not statistically significant $(p<0.177)$ (Table 2$)$.

The power analysis that was carried out for the statistically insignificant variable (inflammatory cell count) revealed that the calculated effect size was 0.389 and the calculated power was $42.7 \%$.

\section{Quantitative RT-PCR}

The highest mean TGF- $\beta$ and vimentin expression values were recorded in the severe periodontitis subgroup (559.60 and 227.60, respectively), whereas the lowest mean values were recorded in the control group (48.84 and 32.18 , respectively). The difference between these 2 groups was statistically significant $(p<0.001)$. On the contrary, there was no significant difference between the control group and the mild periodontitis subgroup (Table 3 ).

The highest mean E-catherin expression value was recorded in the control group, whereas the lowest mean value was recorded in the severe periodontitis subgroup. The difference between these 2 groups was statistically significant $(p<0.001)$. Moreover, there were statistically significant differences between all the studied groups (Table 3).

The correlation analysis between the severity of periodontitis and the expression of both TGF- $\beta 1$ and vimentin revealed a statistically significant positive correlation ( $r=0.895 ; p<0.001$ and $r=0.857 ; p<0.001$, respectively), while E-catherin was found to be significantly negatively correlated $(r=-0.976 ; p<0.001)$ (Table 3).

Table 3. Descriptive statistics regarding the quantitative real-time polymerase chain reaction (RT-PCR) analysis of the expression of TGF- $\beta$, vimentin and E-cadherin, comparisons between the control group and the periodontitis subgroups (ANOVA), and the correlation analysis (Pearson correlation coefficient ( $r$ ))

\begin{tabular}{|c|c|c|c|c|c|c|c|}
\hline \multirow{2}{*}{ Measured items } & \multirow{2}{*}{ Groups } & \multirow{2}{*}{$M \pm S D$} & \multicolumn{2}{|c|}{$95 \% \mathrm{Cl}$} & \multirow{2}{*}{$\begin{array}{c}p \text {-value } \\
\text { (ANOVA) }\end{array}$} & \multicolumn{2}{|c|}{ Correlation } \\
\hline & & & lower bound & upper bound & & $r$ & $p$-value \\
\hline \multirow{4}{*}{ TGF- $\beta 1$} & control group & $48.84^{c} \pm 3.02$ & 45.09 & 52.59 & \multirow{4}{*}{$<0.001^{*}$} & \multirow{4}{*}{0.895} & \multirow{4}{*}{$<0.001^{*}$} \\
\hline & mild periodontitis subgroup & $99.50^{c} \pm 25.33$ & 68.05 & 130.95 & & & \\
\hline & moderate periodontitis subgroup & $299.20^{b} \pm 47.39$ & 240.35 & 358.05 & & & \\
\hline & severe periodontitis subgroup & $559.60^{a} \pm 211.51$ & 296.97 & 822.23 & & & \\
\hline \multirow{4}{*}{ Vimentin } & control group & $32.18^{b} \pm 3.89$ & 27.35 & 37.01 & \multirow{4}{*}{$<0.001^{*}$} & \multirow{4}{*}{0.857} & \multirow{4}{*}{$<0.001^{*}$} \\
\hline & mild periodontitis subgroup & $45.50^{b} \pm 6.03$ & 38.01 & 52.99 & & & \\
\hline & moderate periodontitis subgroup & $105.32^{b} \pm 36.43$ & 60.09 & 150.55 & & & \\
\hline & severe periodontitis subgroup & $227.60^{\mathrm{a}} \pm 76.73$ & 132.32 & 322.88 & & & \\
\hline \multirow{4}{*}{ E-cadherin } & control group & $7.00^{d} \pm 0.16$ & 6.80 & 7.20 & \multirow{4}{*}{$<0.001^{*}$} & \multirow{4}{*}{-0.976} & \multirow{4}{*}{$<0.001^{*}$} \\
\hline & mild periodontitis subgroup & $5.80^{c} \pm 0.56$ & 5.11 & 6.49 & & & \\
\hline & moderate periodontitis subgroup & $3.06^{b} \pm 0.85$ & 2.01 & 4.12 & & & \\
\hline & severe periodontitis subgroup & $1.09^{\mathrm{a}} \pm 0.25$ & 0.78 & 1.39 & & & \\
\hline
\end{tabular}

$\mathrm{Cl}$ - confidence interval; * statistically significant $(p<0.05)$; means sharing the same superscript letter within the same comparison are not significantly different. 


\section{Discussion}

The purpose of this study was to localize and quantify TGF- $\beta 1$ (a cytokine), vimentin (an intermediate filament) and E-cadherin (a cell adhesion molecule) related to the EMT process in human gingival tissue samples from periodontally diseased patients, and to compare them to samples from healthy individuals.

A gradual increase in the thickness of the epithelium was detected in the mild, moderate and severe periodontitis subgroups. The histomorphometric analysis supported this finding, as the highest mean epithelial thickness was recorded in the severe periodontitis gingival tissues, while the lowest was recorded in the control group. This difference was statistically significant $(p<0.028)$.

These findings are in agreement with Tanaskovic Stankovic et al., who reported that an increase in the gingival epithelium was proportional to the severity of inflammation. ${ }^{13}$ The thickening in the inflamed gingival epithelial tissues in the present study could be attributed to a disturbance in tissue homeostasis and the upregulation of inflammatory cytokines, such as interleukin (IL)-1, IL-3, IL-6, and IL-8, which have proliferative effects on human keratinocytes in vitro. ${ }^{14}$

This study reported a reduction in keratinization, especially in the moderate and severe periodontitis subgroups. These findings support the hypothesis that there is a direct relationship between the degree of inflammation and a reduction in keratinization. ${ }^{15}$ In both the moderate and severe periodontitis subgroups, the granular cell layer was flattened and transformed into a superficial cell layer as a means of mechanical protection to compensate for the decreased keratin layer thickness.

The absence of keratohyalin granules observed in the moderate and severe periodontitis subgroups might explain the decrease or absence of keratin detected in both groups. This is because keratohyalin granules play an important role in the synthesis of keratin in keratinized or parakeratinized stratified squamous epithelium. ${ }^{16}$ This point is further supported in this study by the presence of these granules associated with keratin in the gingival specimens of both the mild periodontitis subgroup and the control group.

The severe perinuclear haloing detected throughout the epithelial cell layers in both the moderate and severe periodontitis subgroups is consistent with the findings of Abbass et al.; they reported perinuclear haloing in periodontitis patients when compared with healthy gingiva. ${ }^{17}$ This haloing could be attributed to apoptotic changes that occurred in these cells and the immune system response against severe inflammation. This is supported by the increase in TGF- $\beta 1$ expression in the periodontitis subgroups reported in the present study. Perinuclear haloing was also detected in both the mild periodontitis and healthy tissue samples, but to a lesser degree, as the severity of inflammation diminished.
In the present investigation, the basement membrane of the moderate and severe periodontitis subgroups showed the loss of continuity in some areas, while the basement membrane had a normal appearance in the mild periodontitis subgroup and the normal tissue group. These results are consistent with the findings of Kantarci et al., who reported basement membrane discontinuities in overgrown tissues due to periodontal inflammation when compared to control tissues. ${ }^{18}$ Moreover, these findings clarify the role played by EMT in the pathogenesis of periodontitis, as the loss of basement membrane integrity makes it easier for bacterial pathogens to invade the underlying tissues.

The histomorphometric analysis regarding inflammatory cells determined that the highest mean value was observed in the severe periodontitis subgroup, whereas the lowest mean value was in the control group; however, this difference was not statistically significant $(p<0.177)$. These findings are in agreement with Zekonis et al., who reported higher values for lymphocytes and macrophages in patients with chronic periodontitis than in healthy individuals. ${ }^{19}$ Castro et al. also reported a significant gradual increase in the number of lymphocytes in gingival tissue samples, coinciding with the severity of the disease in chronic periodontitis patients. ${ }^{15}$

The lamina propria in the periodontitis subgroups showed a direct increase in the numbers of blood vessels, correlated with the severity of periodontal disease. An increase in the amount of new vasculature is a common sign of inflammation that is known to be mediated by different inflammatory cytokines, including TGF- $\beta 1$, interleukins and others. ${ }^{20}$ These cytokines have been shown to be involved in the initiation of the EMT process. ${ }^{21}$ The data confirms that there is a correlation between the incidence of EMT and the pathogenesis of inflammatory periodontal disease. This is consistent with the results from a previous study that reported a significant increase in the number of blood vessels in the lamina propria of adult chronic periodontitis specimens when compared to healthy tissues. ${ }^{22}$

The current study reported a significant gradual increase in TGF- $\beta 1$ expression in the mild, moderate and severe periodontitis gingival tissues. These results indicate that there is a direct relationship between the severity of inflammation and the expression of TGF- $\beta 1$ in tissues, which in turn represents the activity of the proposed EMT process. These findings are consistent with those of Mize et al., who deduced that TGF- $\beta 1$ mRNA expression levels were significantly increased in individuals with periodontitis when compared to individuals without periodontitis. ${ }^{23}$ Matarese et al. attributed high TGF- $\beta 1$ levels in their periodontitis patients to the body's protective response against periodontitis. ${ }^{24}$ Potentially, it may be that the biological activities of these growth factors resulted in the insufficient remodeling and perfusion of tooth-supporting tissues, and contributed to periodontal destruction. 
A significant decrease in the transcription of E-cadherin in the mild, moderate and severe periodontitis tissue samples was demonstrated in this study. The downregulation of E-cadherin supports the incidence of EMT during periodontitis, as the epithelial cells lose their cellular junctions. These findings are in agreement with the results from a previous study, which demonstrated the downregulation of E-cadherin expression in the inflamed periodontal tissue in vivo. ${ }^{25}$ Moreover, Arun et al. reported that the virulence factors produced by Porphyromonas gingivalis were capable of decreasing epithelial junctional attachments primarily through the downregulation of E-cadherin. ${ }^{26}$ The authors noted that treating periodontal pockets resulted in a significant upregulation of E-cadherin expression in comparison with diseased sites. ${ }^{26}$

The inverse relationship between E-cadherin and TGF- $\beta 1$ expression detected in the current study is consistent with the findings of Cho et al., who reported in 2010 that E-cadherin antagonized the induction of the TGF- $\beta 1$ gene in hepatic cells. ${ }^{27}$

The present study reported a significant upregulation in vimentin expression in all 3 periodontitis subgroups. The RT-PCR statistical analysis showed that the highest mean value was recorded in the severe periodontitis subgroup, whereas the lowest mean value was recorded in the control group; this difference was statistically significant $(p<0.001)$. These results in addition to the E-cadherin results confirmed that EMT occurs during periodontitis, as the epithelial cells lose their characteristic features and acquire a mesenchymal phenotype. In agreement with these findings, an in vitro study reported that the exposure of H400 keratinocytes to bacterial components revealed the upregulation of vimentin expression. ${ }^{28}$ Also, Scanlon et al. reported a simultaneous upregulation of vimentin expression after stimulation with heat-killed periodontal pathogens and Escherichia coli lipopolysaccharides. ${ }^{29}$ Similar results were obtained in another study, in which 4 epithelial cell lines (hepatocellular carcinoma cell lines) were exposed to lipopolysaccharides. ${ }^{30}$

In the current study, the high expression of vimentin recorded in the periodontitis subgroups could be related to the high expression of TGF- $\beta 1$ also observed in these subgroups. The correlation between TGF- $\beta 1$ and vimentin was investigated by Yoshida et al., who reported that treating mammary epithelial cells with TGF- $\beta 1$ induced the expression of vimentin, which was associated with increased cell invasiveness. ${ }^{31}$ Therefore, it could be concluded that there is a direct positive correlation between TGF- $\beta 1$ and vimentin.

The strategies used for treating periodontitis have not changed much over the last decades. Most of these strategies focus on reducing the plaque biofilm. ${ }^{32}$ In the case of aggressive periodontal disease, antibiotic regimens are used to aid in the elimination of bacterial pathogens. ${ }^{33}$
These approaches do not always provide successful outcomes, and are responsible for local and systemic side effects. Furthermore, there is increased concern about bacterial resistance. A further issue with periodontal disease management is the decolonization of the treated periodontal lesion from bacterial reservoirs within the oral cavity. ${ }^{34}$

To date, few clinical trials have investigated interventions that aim to modulate the host response. Moreover, there is scarce experimental research investigating naturally derived anti-EMT treatment. ${ }^{35}$ The data presented in the current study could provide a potential justification for using anti-EMT agents in managing periodontal disease. Maintaining a functional and intact epithelial barrier would prevent the bacterial invasion of the connective tissues and the subsequent aberrant stimulation of the host response, which is involved in tissue breakdown. Ultimately, the question that needs to be addressed before the potential use of EMT blockers in periodontal disease management is whether EMT is an integral part of the cellular survival mechanism or is a failed component of the healing process.

\section{Conclusions}

The data in this study could potentially provide a basis for using anti-EMT agents in managing periodontal disease, since a significant direct correlation between the severity of periodontal disease and the expression of EMT markers was proven.

\section{ORCID iDs}

Karim Wasfi Wadie (D) https://orcid.org/0000-0002-3336-421X Maha Hassan Bashir (D) https://orcid.org/0000-0002-7833-695X Marwa Magdy Saad Abbass (D) https://orcid.org/0000-0002-6455-7516

\section{References}

1. Eke PI, Thornton-Evans GO, Wei L, Borgnakke WS, Dye BA, Genco RJ. Periodontitis in US adults: National Health and Nutrition Examination Survey 2009-2014. J Am Dent Assoc. 2018;149(7):576-588.e6. doi:10.1016/j.2018.04.023

2. Abbass MMS, Rady D, Radwan IA, et al. The occurrence of periodontal diseases and its correlation with different risk factors among a convenient sample of adult Egyptian population: A cross-sectional study. F1000Res. 2019;8:1740. doi:10.12688/f1000research.20310.2

3. Paster BJ, Boches SK, Galvin JL, et al. Bacterial diversity in human subgingival plaque. J Bacteriol. 2001;183(12):3770-3783. doi:10.1128/JB.183.12.3770-3783.2001

4. Hernández M, Dutzan N, García-Sesnich J, et al. Host-pathogen interactions in progressive chronic periodontitis. J Dent Res. 2011;90(10):1164-1170. doi:10.1177/0022034511401405

5. Kalluri R, Neilson EG. Epithelial-mesenchymal transition and its implications for fibrosis. J Clin Invest. 2003;112(12):1776-1784. doi: $10.1172 / \mathrm{JCl} 200320530$

6. Kalluri R, Weinberg RA. The basics of epithelial-mesenchymal transition. J Clin Invest. 2009;119(6):1420-1428. doi:10.1172/JCI39104

7. Gallimidi AB, Fischman S, Revach $B$, et al. Periodontal pathogens Porphyromonas gingivalis and Fusobacterium nucleatum promote tumor progression in an oral-specific chemical carcinogenesis model. Oncotarget. 2015;6(26), 22613-22623. doi:10.18632/oncotarget.4209 
8. Lamouille S, Xu J, Derynck R. Molecular mechanisms of epithelial -mesenchymal transition. Nat Rev Mol Cell Biol. 2014;15(3):178-196. doi:10.1038/nrm3758

9. Arora H, Madapusi BT, Ramamurti A, Narasimhan M, Periasamy S, Rao SR. Immunohistochemical localization of epithelial mesenchymal transition markers in Cyclosporine A induced gingival overgrowth. J Clin Diagn Res. 2016;10(8):ZC48-ZC52. doi:10.7860/JCDR/2016/20808.8271

10. American Academy of Periodontology Task Force Report on the Update to the 1999 Classification of Periodontal Diseases and Conditions. J Periodontol. 2015;86(7):835-838. doi:10.1902/jop.2015.157001

11. White DA, Tsakos G, Pitts NB, et al. Adult Dental Health Survey 2009: Common oral health conditions and their impact on the population. Br Dent J. 2012;213(11):567-572. doi:10.1038/sj.bdj.2012.1088

12. Suvarna SK, Layton C, Bancroft JD, eds. Bancroft's Theory and Practice of Histological Techniques. $7^{\text {th }}$ ed. London, UK: Churchill Livingstone/Elsevier Health Sciences; 2012.

13. Tanaskovic Stankovic S, Cabunac J, Kanjevac T, Milosavljevic Z. Differential histomorphometric changes in normal and inflamed gingival epithelium. SANAMED. 2016;11(3):197-201. doi:10.5937/sanamed1603197T

14. Yano S, Komine M, Fujimoto M, Okochi H, Tamaki K. Mechanical stretching in vitro regulates signal transduction pathways and cellular proliferation in human epidermal keratinocytes. J Invest Dermatol. 2004;122(3):783-790. doi:10.1111/j.0022-202X.2004.22328.x

15. Castro CE, Koss MA, López ME. Cytological analysis of the periodontal pocket in patients with aggressive periodontitis and chronic periodontitis. Contemp Clin Dent. 2014;5(4):495-500. doi:10.4103/0976-237x.142818

16. Deo PN, Deshmukh R. Pathophysiology of keratinization. J Oral Maxillofac Pathol. 2018;22(1):86-91. doi:10.4103/jomfp.JOMFP_195_16

17. Abbass MM, Korany NS, Salama AH, Dmytryk JJ, Safiejko-Mroczka B. The relationship between receptor for advanced glycation end products expression and the severity of periodontal disease in the gingiva of diabetic and non diabetic periodontitis patients. Arch Oral Biol. 2012;57(10):1342-1354. doi:10.1016/j.archoralbio.2012.06.007

18. Kantarci A, Nseir Z, Kim YS, Sume SS, Trackman PC. Loss of basement membrane integrity in human gingival overgrowth. J Dent Res. 2011;90(7):887-893. doi:10.1177/0022034511404703

19. Zekonis G, Barzdziukaite I, Zekonis J, Sadzeviciene R, Simonyte S, Zilinskas J. Local and systemic immune responses in gingivitis and periodontitis. Cent Eur J Med. 2014;9(5):694-703. doi:10.2478/ s11536-013-0328-5

20. Sanjabi S, Zenewicz LA, Kamanaka M, Flavell RA. Anti-inflammatory and pro-inflammatory roles of TGF-beta, IL-10, and IL-22 in immunity and autoimmunity. Curr Opin Pharmacol. 2009;9(4):447-453. doi:10.1016/j.coph.2009.04.008

21. Landskron $G$, De la Fuente $M$, Thuwajit $P$, Thuwajit $C$, Hermoso MA Chronic inflammation and cytokines in the tumor microenvironment. J Immunol Res. 2014;2014:149185. doi:10.1155/2014/149185

22. Penmetsa GS, Baddam S, Manyam R, Dwarakanath CD. Comparison of the number of gingival blood vessels between type 2 diabetes mellitus and chronic periodontitis patients: An immunohistological study. J Indian Soc Periodontol. 2015;19(2):164-168. doi:10.4103/0972-124x.152105

23. Mize TW, Sundararaj KP, Leite RS, Huang Y. Increased and correlated expression of connective tissue growth factor and transforming growth factor beta 1 in surgically removed periodontal tissues with chronic periodontitis. J Periodontal Res. 2015;50(3):315-319. doi:10.1111/jre.12208

24. Matarese G, Isola G, Anastasi GP, et al. Immunohistochemical analysis of TGF- $\beta 1$ and VEGF in gingival and periodontal tissues: A role of these biomarkers in the pathogenesis of scleroderma and periodontal disease. Int J Mol Med. 2012;30(3):502-508. doi:10.3892/ijmm.2012.1024

25. Abe-Yutori M, Chikazawa T, Shibasaki K, Murakami S. Decreased expression of E-cadherin by Porphyromonas gingivalis-lipopolysaccharide attenuates epithelial barrier function. J Periodontal Res. 2017;52(1):42-50. doi:10.1111/jre.12367

26. Arun R, Hemalatha R, Arun KV, Kumar T. E-cadherin and CD1a expression in gingival epithelium in periodontal health, disease and post-treatment. Indian J Dent Res. 2010;21(3):396-401. doi:10.4103/0970-9290.70811
27. Cho IJ, Kim YW, Han CY, et al. E-cadherin antagonizes transforming growth factor $\beta 1$ gene induction in hepatic stellate cells by inhibiting RhoA-dependent Smad3 phosphorylation. Hepatology. 2010;52(6):2053-2064. doi:10.1002/hep.23931

28. Jing $Y Y$, Han $Z P$, Sun $K$, et al. Toll-like receptor 4 signaling promotes epithelial-mesenchymal transition in human hepatocellular carcinoma induced by lipopolysaccharide. BMC Med. 2012;10:98. doi:10.1186/1741-7015-10-98

29. Scanlon CS, Van Tubergen EA, Inglehart RC, D'Silva NJ. Biomarkers of epithelial-mesenchymal transition in squamous cell carcinoma. J Dent Res. 2013;92(2):114-121. doi:10.1177/0022034512467352

30. Abdulkareem AA, Shelton RM, Landini G, Cooper PR, Milward MR. Potential role of periodontal pathogens in compromising epithelial barrier function by inducing epithelial-mesenchymal transition. J Periodontal Res. 2018;53(4):565-574. doi:10.1111/jre.12546

31. Yoshida K, Saito T, Kamida A, et al. Transforming growth factor- $\beta$ transiently induces vimentin expression and invasive capacity in a canine mammary gland tumor cell line. Res Vet Sci. 2013;94(3):539-541. doi:10.1016/j.rvsc.2012.10.016

32. Newman MG, Takei HH, Klokkevold PR, Caranzza FA, eds. Carranza's Clinical Periodontology. $11^{\text {th }}$ ed. St. Louis, USA: Saunders/Elsevier Health Sciences; 2012.

33. Paraskevas S, Huizinga JD, Loos BG. A systematic review and metaanalyses on C-reactive protein in relation to periodontitis. J Clin Periodontol. 2008;35(4):277-290. doi:10.1111/j.1600-051X.2007.01173.x

34. Gao L, Xu T, Huang G, Jiang S, Gu Y, Chen F. Oral microbiomes: More and more importance in oral cavity and whole body. Protein Cell. 2018;9(5): 488-500. doi:10.1007/s13238-018-0548-1

35. Zhao L, Quan Y, Wang J, Wang F, Zheng Y, Zhou A. Vitamin C inhibit the proliferation, migration and epithelial-mesenchymal-transition of lens epithelial cells by destabilizing HIF-1a. Int J Clin Exp Med. 2015;8(9):15155-15163. 